\title{
Qualitative analysis of latent safety threats uncovered by in situ simulation-based operations testing before moving into a single-family-room neonatal intensive care unit
}

\author{
Rita Dadiz ${ }^{1}$ Julie Riccio ${ }^{1} \cdot$ Kristen Brown ${ }^{1} \cdot$ Paula Emrich $^{1} \cdot$ Beverley Robin ${ }^{2} \cdot$ Jesse Bender $^{3,4}$
}

(c) The Author(s), under exclusive licence to Springer Nature America, Inc. 2020

\begin{abstract}
Objectives We aimed to identify: (a) latent safety threats (LSTs) in a new neonatal intensive care unit (NICU) through simulation-based pre-occupancy operations testing, and (b) LSTs that remained unresolved 1-year post-occupancy.

Study design In this qualitative study, 111 healthcare professionals participated in patient care simulations and debriefings in a new NICU. Debriefing transcripts were inductively analyzed to characterize LSTs. Unresolved LSTs were identified 1 year after NICU occupancy.

Results Thematic saturation was attained after analysis of nine debriefings. Four major themes affecting staff function and patient safety emerged from 305 threats: relay of information, workplace design, patient care processes, and patient family and staff focus. One-year post occupancy, 29 (9\%) LSTs remained unresolved.

Conclusion Team debriefings of simulated patient events uncover LSTs that can largely be resolved before transitioning patient care into a new NICU. Understanding how LSTs interact provides a platform to develop viable strategies to mitigate patient safety risks.
\end{abstract}

\section{Introduction}

As neonatal healthcare focuses on strategies to promote family-centered care, facilitate breastfeeding, improve developmental care, and prevent infection, institutions have redesigned neonatal intensive care units (NICUs) from multiple-patient bays to single-family rooms [1-5]. While the intent is to improve patient care, modifications to the healthcare environment may result in unanticipated changes in patient care processes with the potential of patient harm $[6,7]$.

Rita Dadiz

rita_dadiz@urmc.rochester.edu

1 Department of Pediatrics, University of Rochester Medical Center, Rochester, NY, USA

2 Department of Pediatrics, Rush University Medical Center, Chicago, IL, USA

3 Department of Pediatrics, Mission Hospital, Asheville, NC, USA

4 Warren Alpert Medical School of Brown University, Providence, RI, USA
Identifying potential issues provides an opportunity to correct unrecognized areas of harm before transitioning patient care to new healthcare environments. Patient safety programs have demonstrated the effectiveness of clinical simulations to examine patient care processes, such as resuscitation, management of shock, and central line insertion [8-10]. Testing multiple-patient care processes concurrently in a new healthcare environment enables a greater understanding of how human factors, workflows, and technologies interrelate and lead to latent safety threats (LSTs), which are unrecognized threats to patient safety that may result in patient care compromise and poor outcomes [11].

In situ simulation can effectively identify LSTs in various healthcare environments [6, 8, 10, 12-14]. Immersing teams in patient care simulations improves staff preparation and readiness to function in the new healthcare environment [15]. However, a comprehensive framework is needed to help institutions transitioning into new healthcare environments better understand the breadth and diversity of potential LSTs.

In this study, we conducted in situ simulation-based operations testing in a new single-family-room NICU prior to occupancy using patient scenarios to: (1) uncover and 
describe the different types of LSTs that may impact patient safety and staff function, (2) explore how human factors, workflow processes and new technologies interrelate and present potential patient safety compromise, and (3) identify the LSTs that remained unresolved 1 year after occupancy.

\section{Study design}

\section{Study setting, participants, and approval}

This was a qualitative study of interprofessional team debriefings following immersive in situ simulated patient events. The study was conducted in a new level IV NICU at a tertiary medical center between May 2015 and December 2016, before occupancy. Hospital staff who provided patient care or staff support in the NICU were eligible to participate. Participation was voluntary. The institutional review board at the University of Rochester Medical Center approved the study and waived written consent.

\section{Development of simulation scenarios}

The investigator team consisted of four neonatologists, one neonatal nurse practitioner, and one neonatal nurse who have expertise in conducting team simulations and debriefings for research, quality improvement, and education. From September 2014 to April 2015, prior to the study period, they formed an interprofessional 11-member simulation planning team who performed a needs assessment to identify potential areas of patient safety concerns in the new healthcare environment. The needs assessment included a literature review of simulation-based testing of new healthcare environments $[3,7,16]$, consultations with neonatologists and nurses who led similar transitions into new single-family-room NICUs within the previous 5 years, and discussions with NICU leadership and staff about anticipated challenges.

Using the results of the needs assessment, the simulation planning team developed learning objectives that informed the development of 12 simulation scenarios designed to test the interplay of human factors, workflows, and new technologies (e.g., demonstrate that the emergency staff button signals the appropriate team complement via their mobile communication devices). Five patient scenarios represented "background" activities in the NICU (e.g., preterm infant who requires evaluation for increased abdominal girth). Seven additional scenarios occurred as "unanticipated" events of higher acuity that challenged staff to communicate, allocate resources, and troubleshoot problematic situations (e.g., deployment of two delivery room teams to simultaneous deliveries, resuscitation of a NICU infant, etc.). A representative group of staff piloted the scenarios before they were revised and finalized.

\section{Simulation sessions}

The simulation team adapted a simulation-based operations testing framework developed at the Women and Infants Hospital of Rhode Island [7]. A neighborhood of seven single-family rooms, staff work areas, and supply rooms were staged with mannequins, furniture, monitors, computers, equipment, and supplies. Interprofessional teams of 9-18 individuals were selected by convenience sampling based on their availability to participate in the simulations and debriefings. Staff from other specialties (e.g., anesthesiology, obstetrics, surgery, etc.) participated in relevant scenarios. In addition, trained standardized actors portrayed family members; they were provided scripts and coached on their roles by the simulation team.

The simulation team conducted simulations and debriefings until reaching thematic saturation. Each session was $2-3 \mathrm{~h}$ in duration. Staff were briefed on the goals of participation, and then oriented to the mannequins and the new NICU. The simulation team assigned staff to patients, provided short written patient summaries, and briefed individuals on their infants' scenarios. After this orientation, the simulation team commenced the session by conducting concurrent simulations of the 5 "background" scenarios to create a milieu that modeled the types of routine activities that occur in the NICU, such as examining infants, performing procedures, and updating families. In addition, the simulation team interjected 1 of the 7 higher acuity "unanticipated" scenarios. In some instances, these scenarios required a portion of the NICU team to deploy off the unit and/or work with staff from other disciplines such as obstetrics.

During each $30 \mathrm{~min}$ simulation, the simulation team observed and documented how staff functioned and performed patient care. The simulation team did not communicate with participants during the scenarios, except to provide direction when questions arose or if they deviated significantly from scenario objectives.

\section{Post-simulation team debriefings}

Four investigators with expertise in facilitating postsimulation and post-clinical event debriefings conducted face-to-face semi-structured team debriefings immediately following each simulation session with all participants (60-90 min). The investigators utilized a facilitator's guide that combined the Plus-Delta and 3D (Defusing, Discovering and Deepening) models of debriefing [17, 18]. Prior to use, the facilitator's guide was piloted and iteratively refined. 
Participants, including trained actors, reflected on their simulation experience as a group. They identified challenges with, and opportunities to improve, patient care in the new NICU. Following each debriefing, participants provided feedback on the simulation and debriefing process to help facilitators identify how to improve subsequent sessions. Facilitators incorporated feedback to clarify and add debriefing questions that emerged as important topics for discussion.

All debriefings were audio recorded. With the exception of names, a certified medical transcriptionist transcribed audio recordings verbatim. One investigator reviewed transcriptions to ensure data integrity. LSTs, particularly those identified as serious hazards, were communicated to NICU leadership and hospital administrators immediately.

\section{Data analysis}

Using grounded theory, two paired investigators independently analyzed and coded each debriefing transcript [19]. A codebook was developed and iteratively revised during the data analysis process as investigators reviewed and analyzed the data. Investigators refined and updated the codes with analysis of each subsequent transcript [20]. A third investigator reviewed coded transcripts and verified the codes. When there was discordance amongst investigators on specific codes, they discussed the codes together to find a resolution. If no resolution resulted, they consulted a fourth investigator. Investigators synthesized the codes to identify themes and subthemes of LSTs. Each latent safety threat was categorized as minor, major or serious in scope, based on the potential for harm. LSTs that were likely to result in harm if not resolved were defined as serious; LSTs that had the potential to cause harm if linked with other LSTs were defined as major; and systems inefficiencies with a low probability of causing harm to patients, families or staff were defined as minor. The investigators developed visual aids to illustrate the relationship of emerging themes and subthemes [21]. They maintained an audit trail, which was reviewed by a fifth investigator to mitigate unintended bias and support the trustworthiness of content analysis [22].

\section{Results}

Thematic saturation was reached after staff participated in nine simulations and debriefings in teams ranging in size from 9 to 18 people. Staff $(n=100)$ included 11 attending physicians, 15 trainees (neonatology fellows and pediatric residents), 5 advanced practice providers (APPs), 50 nurses, 5 respiratory therapists, 3 patient care technicians, 2 unit secretaries, 1 radiology technician, 2 operating room

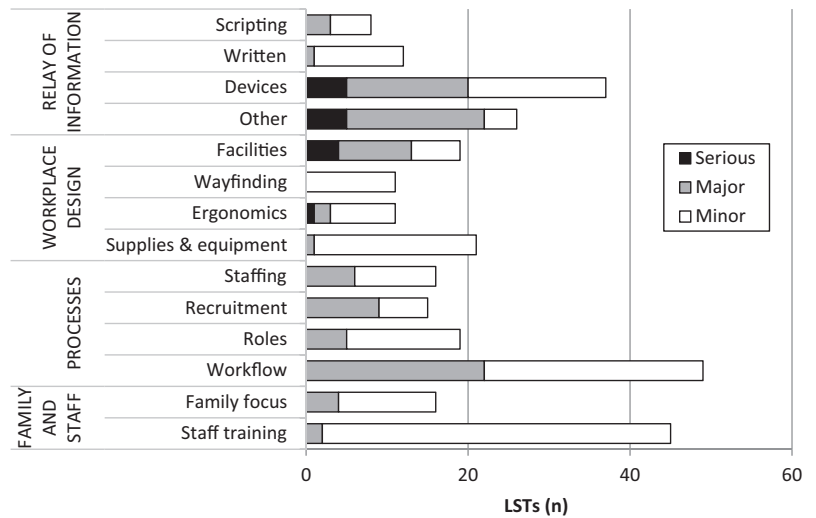

Fig. 1 Latent safety events uncovered from in situ team simulations and debriefings. Qualitative analysis of team debriefings resulted in 305 latent safety threats that were categorized into different themes and subthemes. Latent safety threats ranged in severity from serious to minor.

technicians, 3 public safety officers, and 3 standardized actors. Twenty-one of these participants were from other departments: Anesthesiology, Emergency Medicine, Obstetrics and Gynecology, Imaging Sciences, Patient Safety, Standardized Patient Program and Surgery.

\section{Themes and subthemes}

In-depth analysis of transcribed debriefings revealed 305 LSTs with 4 main themes and 14 subthemes (Fig. 1). The main themes were:

1. Relay of information,

2. Workplace design,

3. Workflow processes, and

4. Family and staff focus.

The majority of identified LSTs were directly or indirectly related to the impact of transitioning patient care from multiple-patient bays to single-family-room design. Because of single-family-room design, the NICU floor plan was elongated and doubled in size. This enlarged footprint required staff to modify their primary mode of communication from in-person conversations to the use of communication devices:

- About communicating deliveries to the delivery room team: "It's impossible to communicate in this space by just looking for somebody. Every morning, you might need to decide [who will be] first responders, second responders. All the delivery room calls will come to the providers' phones. You will need to know there was a delivery, [who went], when they come back, and when they are free to go again. That loop of communication must close when you get back. It might be that we [program] all the providers plus the charge nurse [as a 
group on the phone]. Let's say you go to a delivery, then you text, "I'm back"-that message goes to the whole group on the list, and you don't have to call this person and this person and this person..."

- About using the call buttons in each room to obtain help: "Pushing the call button will be a huge thing, because we don't do this on a typical basis. In the current NICU, you have somebody right there. So, you're now going to have to think, 'All right, I need help, let me push the button.' I'm not going to have time to call somebody on my phone. It's going to be the emergency button."

- About the technology and algorithms used to notify staff about monitor and ventilator alarms: "There are a whole bunch of different thresholds that exist so that multiple people will get the alarms. Let's say your kid is desaturating. If you acknowledge the alarm on the monitor, suction the baby, and he comes right back up, nobody else needs to come rushing in here because you've taken care of it. But if the patient's desaturating and becomes bradycardic, additional alarm thresholds are met, and more people are brought into the loop. I don't know that we've settled on the number of seconds, but the alarm will then start going to other people in the neighborhood and the charge nurse."

In addition, the majority of identified issues were interrelated LSTs. The following debriefing excerpt exemplifies how "workplace design" (i.e., changing from multiplepatient bays to single-family-room design) can affect the "relay of information" and "workflow processes" during patient care. In addition, it demonstrates how in situ simulation helps identify unrecognized LSTs. During this simulation, a bedside nurse activated the code alarm to call for help when an infant became bradycardic, but the APP and physician who were working around the corner in a glass-enclosed charting room did not hear the alarm. The nurse leader assisting the bedside nurse ran to the charting room to alert them. She tried signaling for help through the glass wall, but they were not able to hear her. During the debriefing, the team ultimately realized that no alarm consoles were installed in any of the non-patient work areas to transmit alarm bells.

APP: When we were in [the charting room], we couldn't hear anything...We're like, "Where? What?" You can't hear outside [the charting room] at all. When you were talking, we could not hear you.

Nurse: Can there be communication into the charting room? When you hit the code button, does it go out in the provider area or in the respiratory lab, I wonder?

Physician: It goes overhead, like it does now in the old unit [with] that noise...then the light outside of the [patient's] room lights up.

\section{Latent safety threat mitigation}

Prior to occupancy, identified LSTs were communicated to NICU and hospital leadership. Interprofessional subcommittees collaborated with the simulation team to identify strategies to mitigate and resolve LSTs, and the simulation team conducted subsequent in situ simulations to test the proposed solutions. For example, because staff were not accustomed to the new workflow of activating code alarms instead of calling out for help, patient care during simulations was delayed. Subcommittee members developed guidelines regarding when and how to use the code alarms in the patients' rooms (versus other methods of communication, such as calling out, mobile phones, page operator, etc.). Culture change was reinforced by ongoing participation in simulated patient events before and after transition into the new NICU, as well as in team debriefings of actual patient events after transition into the new NICU. Subcommittee members communicated mitigation strategies to staff via email, monthly newsletters, and staff meetings. They tracked progress on latent safety threat mitigation.

One year after patient care transitioned into the new NICU, the investigators reviewed solutions employed to mitigate LSTs. Of the 305 LSTs identified during the in situ simulations, $276(91 \%)$ were resolved through strategies to improve the relay of information, patient care processes, and when possible, changes to workplace design. Of the remaining 29 LSTs, 10 (3\%) were being actively addressed and $19(6 \%)$ included the implementation of work-around strategies, because no clear solution could be identified.

\section{Conceptual framework}

In-depth analysis of staff experiences during team simulations in the new NICU led to the development of a conceptual framework that illustrates the relationship of the themes and subthemes affecting staff function and patient care/safety (Fig. 2). Serious-to-minor issues resulted from problems related to the relay of information, workplace design, and patient care processes, for which human factors underlie how they interact. As we experienced from the simulations and debriefings, understanding the root causes of these issues helped us identify necessary system changes and staff training needs. Ultimately, appreciating how these themes and subthemes interact resulted in a NICU environment that supports patient safety and staff functioning as high-reliability teams.

\section{Discussion}

Our study revealed that in situ simulation-based operations testing in a representative section of a new single-family-room 


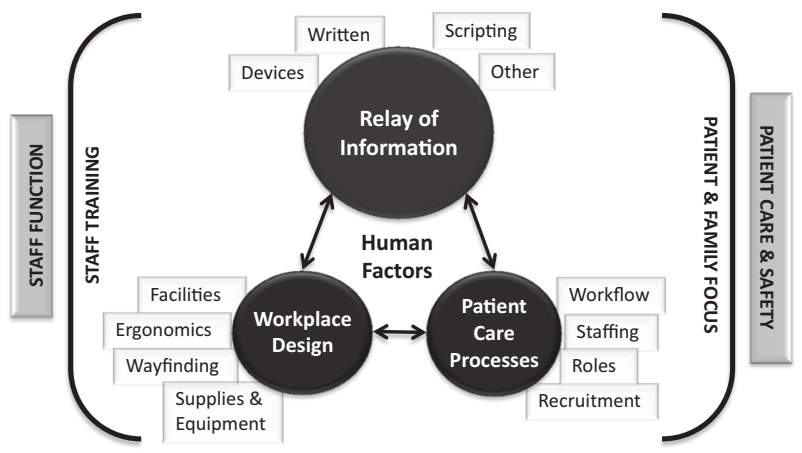

Fig. 2 Framework illustrating the relationship between different latent safety threat themes and subthemes. Qualitative analysis of debriefings led to the development of a conceptual framework that illustrated the relationships between different themes and subthemes of latent safety threats. Relay of information, workplace design, and patient care processes were interconnected. Through greater appreciation of these relationships, institutions may anticipate and mitigate latent safety threats to better support staff function and patient care/ safety.

NICU was instrumental in uncovering LSTs before transitioning patient care into the new healthcare environment. Data analysis highlighted key considerations in the interactions of workplace design, patient care processes, and relay of information that affect staff function and patient care. The majority of identified issues were impacted by single-family-room design, particularly communication failures that resulted from the enlarged NICU footprint and the introduction of new communication devices and workflows. These findings are consistent with Joint Commission and medical malpractice reports citing communication issues as root causes of major patient events that result in patient harm and/or death [23, 24].

Altogether, they highlight the importance of simulationbased operations testing for their discovery, because predicting the shortcomings of a new healthcare environment that result from the interplay of the environment, human factors, and systems integration can be challenging. Involving frontline staff facilitates a more critical examination of these systems to enable greater latent safety threat discovery, because they are more familiar with the intricacies of working in the healthcare environment. Patterson et al. reported that during emergency department simulations, staff identified approximately one-third more LSTs than the simulation facilitators [25]. In addition, conducting simulations before moving into the new healthcare environment allows for planned, systematic evaluations of the healthcare environment without patient distractions, because it can be challenging to fully anticipate and characterize the breadth of LSTs when the environment is "in use" [25, 26].

We found that identified issues were due to interconnected LSTs rather than a sole root cause. In complex clinical environments such as the NICU, weaknesses in systems-based processes, coupled with human factors that trigger mistakes, can cause patient safety compromise, especially if undetected or unresolved LSTs align to result in an overt problem [27]. By identifying LSTs, potential patient compromise can be mitigated through focused strategies, such as implementing new guidelines, revising workflow processes, changing staffing structures, and/or remodeling the physical structure of a new healthcare environment $[27,28]$. Because understanding human factors is integral to the effective function of any complex system, employing simulation-based operations testing that incorporates principles of human-centered design can promote the engagement of frontline staff and unit leadership in the problem-solving process [29].

LSTs identified in our study shared similarities to those reported by some institutions that have conducted in situ simulations prior to transitioning into a new single-familyroom NICU, including communication deficits, missing equipment and supplies, suboptimal staff response times to emergency situations, and staffing issues [3,30]. Although LST similarities exist amongst institutions, there are limited data on the severity, distribution, and interconnected relationships of issues between institutions. Differences between institutions are multifactorial, with variability in workplace design, systems-based processes, and unit-based culture, among others. Regardless of potential differences, our conceptual framework (Fig. 2) and lessons learned from this study can inform the preconstruction design phase for institutions planning to move into a new healthcare environment, avoiding unanticipated downstream effects on patient safety.

In addition to identifying LSTs, simulation-based operations testing improves staff readiness and preparation for transition into new healthcare environments $[12,15,16]$. Immersing staff in team simulations in the new healthcare environment provided opportunities for them to become intimately involved in testing patient care processes. Through post-simulation debriefings and collaboration with interprofessional subcommittees and administrators, staff members additionally participated in the development of solutions to LSTs. This collaborative effort supported staff function and optimized patient care and safety before transitioning patient care into the new healthcare environment.

The majority of issues that persisted 1 year after occupancy of the new healthcare environment were related to communication breakdown due to facility design and limitations of communication devices. Other issues that remained required changes in unit-based culture to adapt new patient care processes such as new workflows, recruitment, and communication strategies. To foster ongoing adaptation of new patient care processes into routine practice, the simulation team continues to conduct ongoing in situ simulations and debriefings so that staff may 
learn and solve problems together in the new healthcare environment.

Several study limitations should be considered in generalizing our results. This study was conducted at a single academic medical center, and the specific types and proportions of identified LSTs may not wholly represent those uncovered in other healthcare environments. In addition, in situ simulations do not fully replicate patient care in the clinical environment. To minimize these limitations, we utilized a variety of clinical scenarios based on a needs assessment, and staff from different professions and disciplines participated to strengthen the yield and quality of team debriefings. Using these strategies, we did not identify any new themes. Conducting post-occupancy team debriefings after in situ simulations and clinical events would further verify the identified themes.

\section{Conclusions}

Immersive in situ simulations and post-simulation debriefings are effective at uncovering LSTs in a new single-familyroom NICU. A conceptual framework that highlights the important interactions between human factors, relay of information, workplace design, and patient care processes can help explore unanticipated risk in the early healthcare environment design stages. Understanding the impact of the new healthcare environment on these factors provides a platform to develop viable solutions that better support staff function and improve patient care. Testing proposed LST mitigation strategies in a simulated environment further strengthens patient safety before transitioning patient care into the new healthcare environment.

For readers who may wish to obtain more information on our findings, please visit: https://nicudesign.nd.edu/.

Author contributions All authors made substantial contributions to the study design, data analysis, and preparation of this paper. They approve this final paper version.

Funding This work was supported in part by the Agency for Healthcare Research and Quality (R18HS023460-01). This article is published as part of a supplement sponsored by Philips.

\section{Compliance with ethical standards}

Conflict of interest $\mathrm{KB}$ has received speaker honoraria from Abbott Nutrition. The remaining authors declared no conflict of interest.

Ethical approval The institutional review board at the University of Rochester Medical Center approved the study and waived written consent.

Publisher's note Springer Nature remains neutral with regard to jurisdictional claims in published maps and institutional affiliations.

\section{References}

1. Altimier L, Phillips R. The neonatal integrative developmental care model: advanced clinical applications of the seven core measures for neuroprotective family-centered developmental care. Newborn Infant Nurs Rev. 2016;16:230-44.

2. White RD. The newborn intensive care unit environment of care: how we got here, where we're headed, and why. Semin Perinatol. 2011;35:2-7.

3. Walsh WF, McCullough KL, White RD. Room for improvement: nurses' perceptions of providing care in a single room newborn intensive care setting. Adv Neonatal Care. 2006;6:261-70.

4. Domanico R, Davis DK, Coleman F, Davis BO. Documenting the NICU design dilemma: comparative patient progress in open-ward and single family room units. J Perinatol. 2011;31:281-8.

5. Lester BM, Hawes K, Abar B, Sullivan M, Miller R, Bigsby R, et al. Single-family room care and neurobehavioral and medical outcomes in preterm infants. Pediatrics. 2014;134:754-60.

6. Villamaria FJ, Pliego JF, Wehbe-Janek H, Coker N, Rajab MH, Sibbitt $\mathrm{S}$, et al. Using simulation to orient code blue teams to a new hospital facility. Simul Healthc. 2008;3:209-16.

7. Bender GJ. In situ simulation for systems testing in newly constructed perinatal facilities. Semin Perinatol. 2011;35:80-3.

8. Knight P, MacGloin H, Lane M, Lofton L, Desai A, Haxby E, et al. Mitigating latent threats identified through an embedded in situ simulation program and their comparison to patient safety incidents: a retrospective review. Front Pediatr. 2017;5:281.

9. Wetzel EA, Lang TR, Pendergrass TL, Taylor RG, Geis GL. Identification of latent safety threats using high-fidelity simulation-based training with multidisciplinary neonatology teams. Jt Comm J Qual Patient Saf. 2013;39:268-73.

10. Kobayashi L, Shapiro MJ, Sucov A, Woolard R, Boss RM 3rd, Dunbar J, et al. Portable advanced medical simulation for new emergency department testing and orientation. Acad Emerg Med. 2006;13:691-5.

11. Halamek LP. Editorial: Bringing latent safety threats out into the open. Jt Comm J Qual Patient Saf. 2013;39:267.

12. Adler MD, Mobley BL, Eppich WJ, Lappe M, Green M, Mangold $\mathrm{K}$. Use of simulation to test systems and prepare staff for a new hospital transition. J Patient Saf. 2018;14:143-7.

13. Wheeler DS, Geis G, Mack EH, LeMaster T, Patterson MD. Highreliability emergency response teams in the hospital: improving quality and safety using in situ simulation training. BMJ Qual Saf. 2013;22:507-14.

14. Geis GL, Pio B, Pendergrass TL, Moyer MR, Patterson MD. Simulation to assess the safety of new healthcare teams and new facilities. Simul Healthc. 2011;6:125-33.

15. Bender GJ, Maryman JA. Clinical macrosystem simulation translates between organizations. Simul Healthc. 2018;13:96-106.

16. Carlson B, Walsh S, Wergin T, Schwarzkopf K, Ecklund S. Challenges in design and transition to a private room model in the neonatal intensive care unit. Adv Neonatal Care. 2006;6:271-80.

17. Helminski L. Total quality in instruction: a systems approach. In Roberts HV, editor. Academic initiatives in total quality for higher education. Milwaukee, WI: ASQC Quality Press; 1995.

18. Zigmont JJ, Kappus LJ, Sudikoff SN. The 3D model of debriefing: defusing, discovering, and deepening. Semin Perinatol. 2011;35:52-8.

19. Charmaz K. Constructing grounded theory: a practical guide through qualitative analysis. Newbury Park, CA: Sage Publishing; 2006.

20. Corbin JS. Basics of qualitative research: techniques and procedures for developing grounded theory, 3rd ed. Thousand Oaks, CA: Sage Publishing; 2008. 
21. Borkan J. Immersion/crystallization: doing qualitative research, 2nd ed. Thousand Oaks, CA: Sage Pubilications; 1999.

22. Miles M, Huberman M. Qualitative data analysis: an expanded sourcebook, 2nd ed. Thousand Oaks, CA: Sage Pubilications; 1994.

23. The Joint Commission. Preventing infant death and injury during delivery. Jt Comm Perspect. 2004;24:14-5.

24. Keohane CA, Dwyer K, Boulanger J, Zigmont K, Babayan A, Cushing E, et al. Partnering with a medical malpractice insurer to improve patient safety and decrease risk. J Perinat Neonatal Nurs. 2018;32:66-71.

25. Patterson MD, Geis GL, Falcone RA, LeMaster T, Wears RL. In situ simulation: detection of safety threats and teamwork training in a high risk emergency department. BMJ Qual Saf. 2013;22:468-77.
26. Braithwaite J, Wears R, Hollnagel E. Resilient health care, volume 3: reconciling work-as-imagined and work-as-done. Boca Raton, FL: CRC Press; 2017.

27. Reason J. Human error: models and management. BMJ. 2000;320:768-70.

28. Agency for Healthcare Research and Quality. Patient safety primer: systems approach. Rockville, MD: AHRQ patient safety network. https://psnet.ahrq.gov/primer/systems-approach. Accessed 21 Oct 2019.

29. Searl MM, Borgi L, Chemali Z. It is time to talk about people: a human-centered healthcare system. Health Res Policy Syst. 2010;8:35.

30. Bender J, Shields R, Kennally K. Transportable enhanced simulation technologies for pre-implementation limited operations testing: neonatal intensive care unit. Simul Healthc. 2011;6:204-12. 